\title{
Mucous Membrane Pemphigoid-Associated Malignancies: Case Series and a Brief Overview of the Literature
}

\author{
Michelangelo La Placa ${ }^{1}$, Riccardo Balestri ${ }^{1}$, Federico Tartari ${ }^{1}$, Andrea Sechi ${ }^{1}$, Francesca Ferrara ${ }^{1}$, \\ Camilla Loi ${ }^{1}$, Annalisa Patrizi ${ }^{1}$, Federico Bardazzi ${ }^{1}$
}

1 Dermatology Division, Department of Experimental, Diagnostic and Specialty Medicine, University of Bologna, Bologna, Italy

Key words: mucous membrane pemphigoid, malignancy, autoimmune bullous disorders, paraneoplastic pemphigus, laminin-332, blistering diseases, laminin-5, anti-epiligrin, cicatricial pemphigoid, adenocarcinoma

Citation: La Placa M, Balestri R, Tartari F, Sechi A, Ferrara F, Loi C, Patrizi A, Bardazzi F. Mucous membrane pemphigoid-associated malignancies: Case series and a brief overview of the literature. Dermatol Pract Concept. 2019;9(2):119-125. DOI: https://doi.org/10.5826/ dpc.0902a07

Accepted: February 6, 2019; Published: April 30, 2019

Copyright: @2019 La Placa et al. This is an open-access article distributed under the terms of the Creative Commons Attribution License, which permits unrestricted use, distribution, and reproduction in any medium, provided the original author and source are credited.

Funding: None.

Competing interests: The authors have no conflicts of interest to disclose.

Authorship: All authors have contributed significantly to this publication.

Corresponding author: Michelangelo La Placa, MD, Dermatology Division, Department of Experimental, Diagnostic and Specialty Medicine, University of Bologna, Via Massarenti 1, 40138, Bologna, Italy. Email: michelangelo.laplaca@unibo.it

ABSTRACT Background: Mucous membrane pemphigoid (MMP) is a heterogeneous group of blistering disorders affecting the mucosae with or without skin involvement, characterized by the presence of autoantibodies to components of the basement membrane zone, including the bullous pemphigoid antigen BP180 and $\beta 4$ integrin. Current literature has shown that a minority of patients present circulating antibodies to laminin-332 and this population seems to be associated with a relatively high risk of malignancy.

Objective: To present our personal case series of patients with MMP-associated malignancy from a dermatology university hospital.

Methods: Twenty-two patients affected by MMP were seen in the period between 2001 and 2016; in 4 patients $(18 \%)$ an associated cancer was detected.

Results: These patients were 2 men and 2 women, with a mean age of 69.7 years (range, 48-83). The associated malignancies included a breast cancer, a pancreatic adenocarcinoma, a metastatic laryngeal carcinoma, and a hepatic carcinoma. All patients had negative results for both BP180 and laminin-332 autoantibodies.

Conclusion: We confirm that MMP patients have a relatively high possibility of developing a solid cancer, but the autoantibody detection is not mandatory and is probably correlated with the severity of the disease.

\section{Introduction}

Mucous membrane pemphigoid (MMP), formerly cicatricial pemphigoid, is a rare heterogeneous group of autoimmune subepithelial blistering disorders involving the mucous mem- branes and occasionally the skin with a chronic course and tendency toward scarring [1]. Previously, other names have been used to describe these conditions, including benign MMP, anti-laminin-5 cicatricial pemphigoid, oral pemphigoid, and ocular pemphigoid. Because it is difficult to clini- 
cally distinguish the various MMP subgroups, the collective term MMP is now accepted [2].

MMP commonly affects the oral mucosa, but ocular and nasal epithelia, the first aerodigestive tract and the genitals may be involved. Cutaneous involvement may be absent or limited. MMP usually affects patients aged between 60 and 80 years, with a female prevalence [1-3]. The disease is mainly controlled with corticosteroids and other immunosuppressive drugs, including cyclosporine, mycophenolate, cyclophosphamide, and azathioprine [3].

Most of the MMP autoantibody profile shares the same target antigens of other autoimmune blistering diseases from the pemphigoid group and paraneoplastic pemphigus, namely circulating IgG and/or IgA autoantibodies targeting the basement membrane zone (BMZ) components, including the bullous pemphigoid antigens BP180 and BP230, and the $\beta 4$ integrin [1-6]. Current evidence demonstrates that a minority of MMP patients with autoantibodies to IgG antilaminin-332 (formerly anti-laminin-5 or anti-epiligrin) have an increased relative risk of cancer [1,7]. Previously, this subgroup was named anti-epiligrin cicatricial pemphigoid (AECP), to differentiate it from the majority of cicatricial pemphigoid patients without these specific autoantibodies. Finally, there is no report of MMP-associated malignancies with autoantibodies to BP180 [1,2,8-14].

We present a case series of MMP patients with associated malignancies and a brief overview of the current literature.

\section{Case Series}

During the period between 2001 and 2016, at the Department of Dermatology at the University of Bologna, we diagnosed and followed up 22 patients affected by MMP. From this group, we selected 4 individuals (18\%) who developed a solid tumor (a breast cancer, a pancreatic adenocarcinoma, a metastatic laryngeal carcinoma, and a hepatocarcinoma) before the MMP diagnosis or during the MMP follow-up. They were 2 men and 2 women, with a mean age of 69.7 years (range, 48-83). In general, our patients had mainly oral and/or genital mucous involvement. Only patient 2 had concomitant skin lesions, characterized by blisters and erosions on the trunk and limbs, and patient 4 had exclusive conjunctival involvement, undergoing a complete loss of eyelashes due to synechiae. Cancer detection was preceding in case 1 (diagnosed 1 year before MMP) and metachronous in the remaining cases: 1 year after MMP in cases 2 and 4, and 3 years after in case 3. All patients' data, treatment, and followup are summarized in Table 1. Another patient, a 73-year-old woman, received the diagnosis of breast cancer 6 years preceding MMP occurrence. She is still under follow-up in our

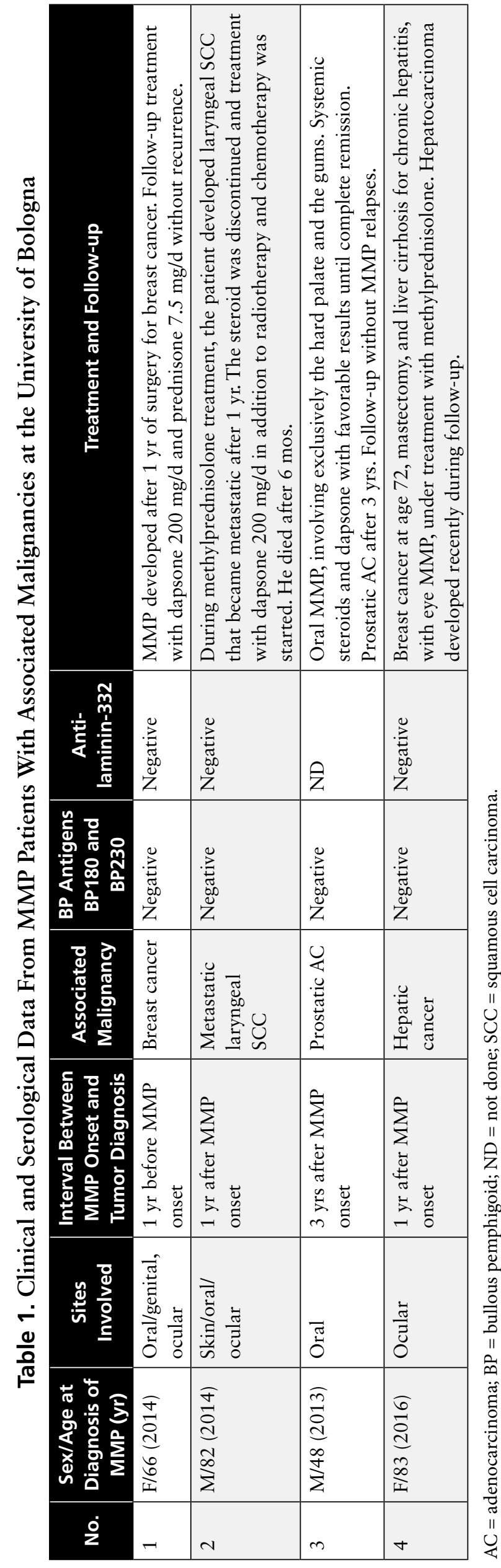




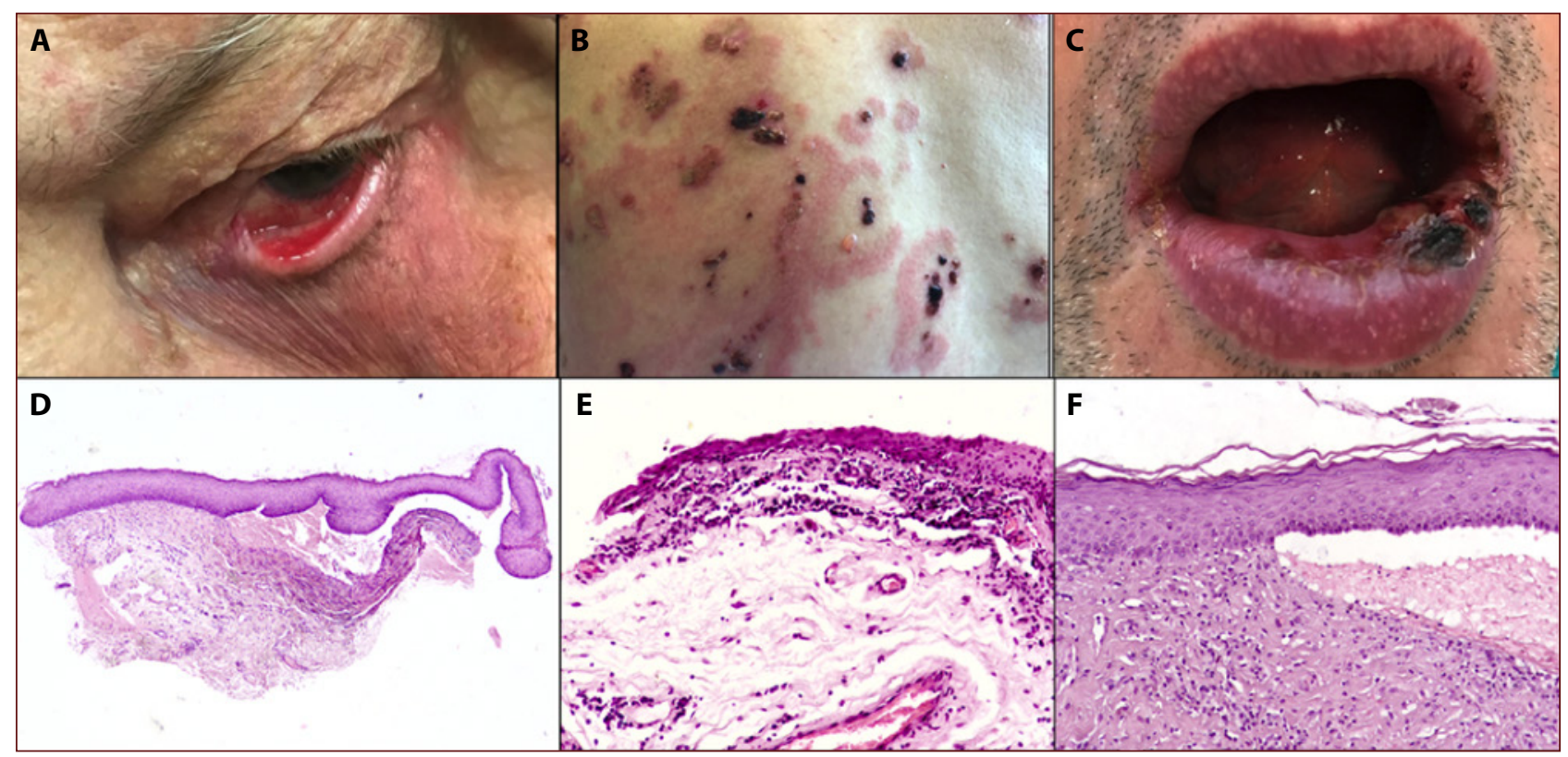

Figure 1. (A) Ocular MMP with conjunctival involvement, with symblepharon formation. (B) Polymorphic manifestation of MMP with cutaneous involvement, characterized by tense, serous, or hemorrhagic bullae. (C) Oral MMP with hemorrhagic crusting and lip erosions. (D) Low magnification of subepidermal blister with inflammatory cell infiltrate (hematoxylin and eosin [H\&E], x4). (E,F) MMP inflammatory infiltrate of the upper dermis, with numerous eosinophils (H\&E, $\times 10$; H\&E, ×20). [Copyright: $\odot 2019$ La Placa et al.]

clinic, but was excluded from the study because of the long interval between cancer occurrence and the MMP diagnosis.

An MMP diagnosis was made by combining the clinical and histopathological findings. In particular, clinical findings included active mucous membrane involvement characterized by blisters and/or erosions. In addition, direct and indirect immunofluorescence from perilesional mucosa revealed a linear deposition of IgG and/or C3 at the BMZ and circulating IgG anti-BMZ autoantibodies, respectively (Figure 1). Immunoblot analysis was negative for anti-BP180 and anti-BP230 autoantibodies, as well as anti-purified human laminin-332.

The investigation described was carried out on residual biopsy sections following diagnostic analysis during the course of institutional diagnostic services, and the study was exempted from institutional review board review.

\section{Discussion}

MMP is an autoimmune bullous disorder with predominant or exclusive mucosal involvement. As reported for paraneoplastic pemphigus and bullous pemphigoid, MMP is a pathogenetic example of cell-mediated immunity involvement, characterized by the presence of different autoantibody responses to basement membrane antigens, including the BP180 and BP230, or $\beta 4$ integrin [3]. Moreover, whereas most of MMP patients share BP180 autoantigen and oral or cutaneous involvement [12], a minority of individuals express laminin-332 antigen, which is a glycoprotein that interacts with other BMZ molecules to stabilize the extracel- lular matrix [1,2,6-9]. These individuals cannot be distinguished clinically from those with other variants of MMP [10]. Because it has been reported that these patients with anti-laminin-332 have an increased relative risk of developing cancer, in particular adenocarcinoma, prompt diagnosis and treatments are crucial [8-12]. Various pathogenetic hypotheses have been put forward, including the theory that tumor cells secrete laminin-332 with the consequent loss of keratinocyte adhesion and blister formation [10].

A literature search revealed several case reports and retrospective cohort studies regarding MMP and malignancy [8,12-36]. In particular, Egan et al described a cohort of 35 patients affected by AECP followed up in a period of 12 years; 10 patients $(28.6 \%)$ developed a solitary cancer [8]. Eight of these patients had cancer after the onset of AECP, most within 12 to 14 months, and all deaths were cancer-related. The associated malignancies included 3 lung, 3 stomach, 2 colon, and 2 endometrial cancers. They estimated a relative risk of $6.8 \%$ for solid cancer, but $15.4 \%$ if diagnosed within the first year of blister formation. They also documented the short interval occurring between the onset of AECP and cancer (average 14 months). This was the first cohort study regarding MMP and associated malignancies. Later, Letko et al have published a retrospective study of 79 patients affected by MMP [11]. Only in 3 patients was a cancer associated with MMP; a breast ductal carcinoma in situ, a lung squamous cell carcinoma, and a colon adenocarcinoma. Three other oncology patients had been excluded from the study because the cancer diagnosis preceded the MMP (13, 


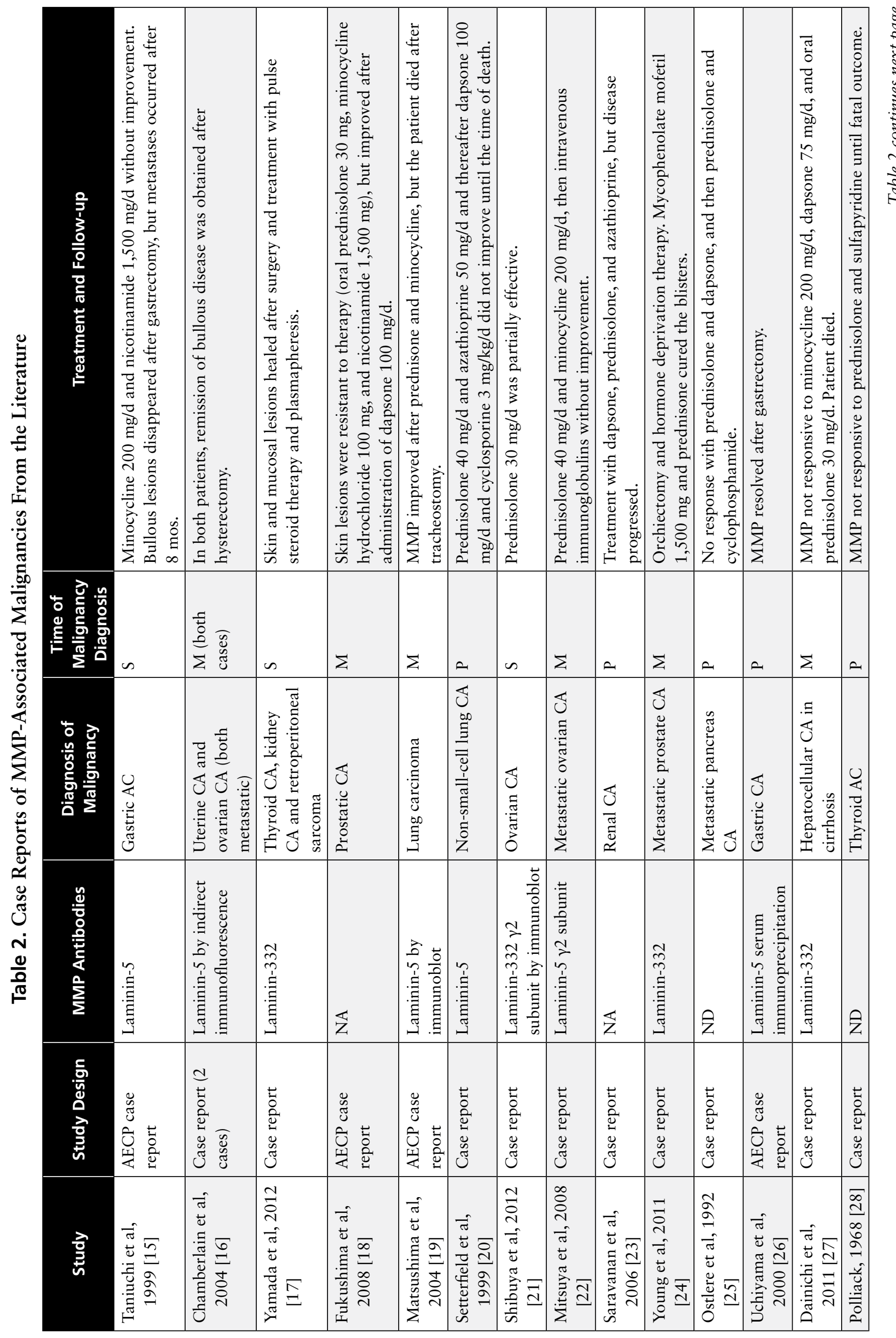




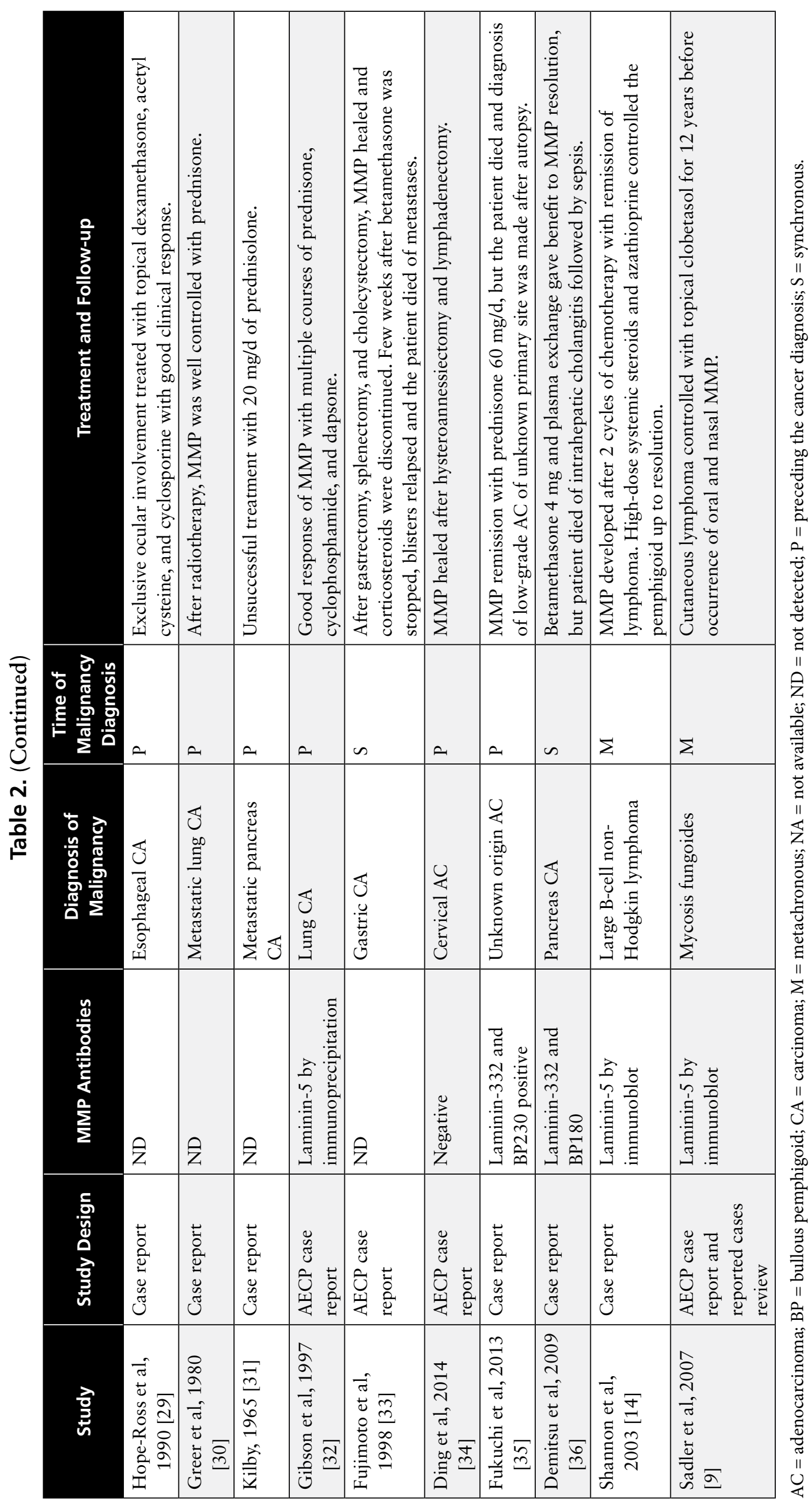


5 , and 4 years, respectively). In another report, Sadler et al described a patient with mycosis fungoides treated for 12 years with topical clobetasol ointment who developed oral and nasal erosions; histopathology led to the diagnosis of AECP [9]. This is the first association between MMP and lymphoma. Moreover, the authors provided a summary overview of all MMP (or AECP) cancer-associated cases published until 2007, including a letter from Shannon and colleagues [14], reporting a case of cicatricial pemphigoid and non-Hodgkin lymphoma. More recently, Bernard and colleagues analyzed a large cohort of $154 \mathrm{MMP}$ patients, and an associated neoplasia was found in $18(11.7 \%)$ of them [13]. In this French study, the prevalence and significance of anti-laminin-332 was analyzed, showing that the presence of these antibodies is directly correlated with the severity of the disease, but not exclusively with the presence of a neoplasm. In fact, of these 18 patients, they showed that only $2(6.4 \%)$ were laminin-332 positive, while 16 (13\%) were laminin-332 negative, concluding that this frequency does not differ from that of the general population in the same age range. It is noteworthy, finally, that in most of these cases the interval between cancer detection and MMP occurrence was very long (more than 8 years), and the cancer diagnosis frequently preceded the MMP occurrence.

On the other hand, case reports published to date include $23 \mathrm{MMP}$ or AECP patients with 25 solid tumors, in particular lung, gastric, ovarian, cervical, and pancreatic adenocarcinomas; and renal, prostatic, thyroid, hepatocellular, and esophageal carcinomas [8,14-36]. In addition, a case of large B-cell non-Hodgkin lymphoma and a case of mycosis fungoides are cited above [8,14]. Although it is sometimes impossible to clarify the time of cancer detection, in 16 cases the diagnosis of MMP preceded the tumor diagnosis or was synchronous (11 and 5 cases, respectively), whereas in 9 patients the bullous disorder occurred after the cancer detection (metachronous) (Table 2).

Determining a true paraneoplastic syndrome is often impossible. In fact, most neoplasms are initially occult and asymptomatic. In particular, a true paraneoplastic syndrome comprises the contemporaneous presence (or detection) of both dermatosis and cancer, with resolution of symptoms after cancer healing, and possible recurrence after cancer relapse. Therefore, the term "paraneoplastic" is used only for a subtype of pemphigus, while the term "associated malignancies" must be preferred in the other autoimmune blistering disorders, including MMP [37].

In our case series, 3 patients developed the malignancy 1 to 3 years after the MMP diagnosis. A woman had breast cancer 1 year before MMP occurrence. Although 1 of our patients died from metastases 1.5 year after MMP diagnosis (case 2), the others cleared their bullous disease after cancer removal and are still under follow-up (range, 2-5 years) in our clinic without recurrences.

\section{Conclusions}

The clinical importance of determining specific antibodies in autoimmune blistering disorders is well known. For example, the presence of BP180 and/or BP230 in bullous pemphigoid may represent an active disease also in the absence of clinical symptoms [38]. In this regard, various studies demonstrate the correlation between laminin-332 MMP and cancer, underscoring the importance of immunological diagnosis of these autoantibodies $[7,10]$.

On the other hand, and in accordance with the recent study by Bernard and coworkers [13], our data demonstrate the relatively frequent association of MMP with a solid cancer, but no significant correlation with autoantibody detection. However, it is possible that laminin-332 reactivity is correlated with the severity of the disease, but needs to be determined by further studies.

\section{References}

1. Kartan S, Shi VY, Clark AK, Chan LS. Paraneoplastic pemphigus and autoimmune blistering diseases associated with neoplasm: characteristics, diagnosis, associated neoplasms, proposed pathogenesis, treatment. Am J Clin Dermatol. 2017;18(1):105-126.

2. Chan LS, Ahmed AR, Anhalt GJ, et al. The first international consensus on mucous membrane pemphigoid: definition, diagnostic criteria, pathogenic factors, medical treatment, and prognostic indicators. Arch Dermatol. 2002;138 (3):370-379.

3. Broussard KC, Leung TG, Moradi A, Thorne JE, Fine JD. Autoimmune bullous diseases with skin and eye involvement: cicatricial pemphigoid, pemphigus vulgaris, and pemphigus paraneoplastica. Clin Dermatol. 2016;34(2):205-213.

4. Anhalt GJ, Kim SC, Stanley JR, et al. Paraneoplastic pemphigus: an autoimmune mucocutaneous disease associated with neoplasia. N Engl J Med. 1990;323(25):1729-1735.

5. Mutasim DF, Pelc NJ, Anhalt GJ. Paraneoplastic pemphigus, pemphigus vulgaris, and pemphigus foliaceous. Clin Dermatol. 1993;11(3):173-181.

6. Bhol KC, Colon JE, Ahmed AR. Autoantibody in mucous membrane pemphigoid binds to an intracellular epitope on human beta 4 integrin and causes basement membrane zone separation in oral mucosa in an organ culture model. J Invest Dermatol. 2003;120(4):701-702.

7. Lazarova Z, Salato VK, Lanschuetzer CM, Janson M, Fairley JA, Yancey KB. IgG anti-laminin-332 autoantibodies are present in a subset of patients with mucous membrane, but not bullous, pemphigoid. J Am Acad Dermatol. 2008;58(6):951-958.

8. Egan CA, Lazarova Z, Darling TN, Yee C, Coté T, Yancey KB. Anti-epiligrin cicatricial pemphigoid and relative risk of cancer. Lancet. 2001;357 (9271):1850-1851.

9. Sadler E, Lazarova Z, Sarasombath P, Yancey KB. A widening perspective regarding the relationship between anti-epiligrin cicatricial pemphigoid and cancer. J Dermatol Sci. 2007;47(1):1-7. 
10. Egan CA, Lazarova Z, Darling TN, Yee C, Yancey KB. Antiepiligrin cicatricial pemphigoid: clinical findings, immunopathogenesis, and significant associations. Medicine (Baltimore). 2003;82(3):177-186.

11. Letko E, Gürcan HM, Papaliodis GN, Christen W, Foster CS, Ahmed AR. Relative risk for cancer in mucous membrane pemphigoid associated with antibodies to the beta4 integrin subunit. Clin Exp Dermatol. 2007;32(6):637-641.

12. Cozzani E, Di Zenzo G, Calabresi V, et al. Autoantibody profile of a cohort of 78 Italian patients with mucous membrane pemphigoid: correlation between reactivity profile and clinical involvement. Acta Derm Venereol. 2016;96(6):768-773.

13. Bernard P, Antonicelli F, Bedane C, et al. Prevalence and clinical significance of anti-laminin 332 autoantibodies detected by a novel enzyme-linked immunosorbent assay in mucous membrane pemphigoid. JAMA Dermatol. 2013;149(5):533-540.

14. Shannon JF, Mackenzie-Wood A, Wood G, Goldstein D. Cicatricial pemphigoid in non-Hodgkin's lymphoma. Intern Med J. 2003;33(8):396-397.

15. Taniuchi K, Takata M, Matsui C, et al. Antiepiligrin (laminin 5) cicatricial pemphigoid associated with an underlying gastric carcinoma producing laminin 5. Br J Dermatol. 1999;140(4):696-700.

16. Chamberlain AJ, Cooper SM, Allen J, et al. Paraneoplastic immunobullous disease with an epidermolysis bullosa acquisita phenotype: two cases demonstrating remission with treatment of gynaecological malignancy. Australas J Dermatol. 2004;45(2):136-139.

17. Yamada H, Nobeyama Y, Matsuo K, et al. A case of paraneoplastic pemphigus associated with triple malignancies in combination with antilaminin-332 mucous membrane pemphigoid. $\mathrm{Br}$ J Dermatol. 2012;166(1):230-231.

18. Fukushima S, Egawa K, Nishi H, et al. Two cases of anti-epiligrin cicatricial pemphigoid with and without associated malignancy. Acta Derm Venereol. 2008;88(5):484-487.

19. Matsushima $S$, Horiguchi $Y$, Honda T, et al. A case of anti-epiligrin cicatricial pemphigoid associated with lung carcinoma and severe laryngeal stenosis: review of Japanese cases and evaluation of risk for internal malignancy. J Dermatol. 2004;31(1):10-15.

20. Setterfield J, Shirlaw PJ, Lazarova Z, et al. Paraneoplastic cicatricial pemphigoid. Br J Dermatol. 1999;141(1):127-131.

21. Shibuya T, Komatsu S, Takahashi I, et al. Mucous membrane pemphigoid accompanied by ovarian cancer: a case with autoantibodies solely against $\gamma(2)$-subunit of laminin-332. J Dermatol. 2012;39(10):882-884.

22. Mitsuya J, Hara H, Ito K, Ishii N, Hashimoto T, Terui T. Metastatic ovarian carcinoma-associated subepidermal blistering disease with autoantibodies to both the p200 dermal antigen and the gamma 2 subunit of laminin 5 showing unusual clinical features. Br J Dermatol. 2008;158(6):1354-1357.

23. Saravanan K, Baer ST, Meredith A, Dyson A, von der Werth J. Benign mucous membrane pemphigoid of the upper aero-digestive tract: rare paraneoplastic syndrome presentation in renal cell carcinoma. J Laryngol Otol. 2006;120(3):237-239.
24. Young AL, Bailey EE, Colaço SM, Engler DE, Grossman ME. Anti-laminin-332 mucous membrane pemphigoid associated with recurrent metastatic prostate carcinoma: hypothesis for a paraneoplastic phenomenon. Eur J Dermatol. 2011;21(3):401-404.

25. Ostlere LS, Branfoot AC, Staughton RC. Cicatricial pemphigoid and carcinoma of the pancreas. Clin Exp Dermatol. 1992;17(1):67-68.

26. Uchiyama K, Yamamoto Y, Taniuchi K, Matsui C, Fushida Y, Shirao Y. Remission of antiepiligrin (laminin-5) cicatricial pemphigoid after excision of gastric carcinoma. Cornea. 2000;19(4):564566.

27. Dainichi T, Hirakawa Y, Ishii N, et al. Mucous membrane pemphigoid with autoantibodies to all the laminin 332 subunits and fatal outcome resulting from liver cirrhosis and hepatocellular carcinoma. J Am Acad Dermatol. 2011;64(6):1199-1200.

28. Polliack A. Benign mucous membrane pemphigoid with laryngeal stenosis in a patient with thyroid carcinoma. Arch Pathol. 1968;86(1):48-51.

29. Hope-Ross M, Benedict-Smith A, Hillery M, Mullaney P, Condon P, Collum LM. Ocular cicatricial pemphigoid and oesophageal carcinoma. Acta Ophthalmol (Copenh). 1990;68(3):361-363.

30. Greer KE, Beacham BE, Askew FC Jr. Benign mucous membrane pemphigoid in association with internal malignancy. Cutis. 1980;25(2):183-185.

31. Kilby PE. Carcinoma of the pancreas presenting with "benign mucous membrane pemphigoid.” Cancer. 1965;18:847-850.

32. Gibson GE, Daoud MS, Pittelkow MR. Anti-epiligrin (laminin 5) cicatricial pemphigoid and lung carcinoma: coincidence or association? Br J Dermatol. 1997;137(5):780-782.

33. Fujimoto W, Ishida-Yamamoto A, Hsu R, et al. Anti-epiligrin cicatricial pemphigoid: a case associated with gastric carcinoma and features resembling epidermolysis bullosa acquisita. $\mathrm{Br} \mathrm{J}$ Dermatol. 1998;139(4):682-687.

34. Ding DC, Chu TY, Hsu YH. Remission of anti-epiligrin cicatricial pemphigoid after excision of cervical adenocarcinoma. J Cutan Pathol. 2014;41(8):692-693.

35. Fukuchi O, Suko A, Matsuzaki H, et al. Anti-laminin-332 mucous membrane pemphigoid with autoantibodies to $\alpha 3, \beta 3$ and $\gamma 2$ subunits of laminin-332 as well as to BP230 and periplakin associated with adenocarcinoma from an unknown primary site. J Dermatol. 2013;40(1):61-62.

36. Demitsu T, Yoneda K, Iida E, et al. A case of mucous membrane pemphigoid with IgG antibodies against all the $\alpha 3, \beta 3$ and $\gamma 2$ subunits of laminin-332 and BP180 C-terminal domain, associated with pancreatic cancer. Clin Exp Dermatol. 2009;34(8):e992e994.

37. Balestri R, Magnano M, La Placa M, et al. Malignancies in bullous pemphigoid: a controversial association. J Dermatol. 2016;43(2):125-133.

38. Schmidt E, Obe K, Bröcker EB, Zillikens D. Serum levels of autoantibodies to BP180 correlate with disease activity in patients with bullous pemphigoid. Arch Dermatol. 2000;136(2):174-178. 